\title{
Optical Parametric Oscillators and Amplifiers for Airborne and Spaceborne Active Remote Sensing of $\mathrm{CO} 2$ and $\mathrm{CH} 4$
}

\author{
Andreas Fix*, Christian Büdenbender, Martin Wirth, Mathieu Quatrevalet, Axel Amediek, \\ Christoph Kiemle, and Gerhard Ehret
}
Deutsches Zentrum für Luft- und Raumfahrt (DLR), Institut für Physik der Atmosphäre, 82234 Oberpfaffenhofen, Germany

\begin{abstract}
Carbon dioxide (CO2) and methane (CH4) are the most important of the greenhouse gases that are directly influenced by human activities. The Integrated Path Differential Absorption (IPDA) lidar technique using hard target reflection in the near IR $(1.57 \mu \mathrm{m}$ and $1.64 \mu \mathrm{m})$ to measure the column-averaged dry air mixing ratio of $\mathrm{CO} 2$ and $\mathrm{CH} 4$ with high precision and low bias has the potential to deliver measurements from space and air that are needed to understand the sources and sinks of these greenhouse gases. $\mathrm{CO} 2$ and $\mathrm{CH} 4$ IPDA require tunable laser sources at $1.57 \mu \mathrm{m}$ and $1.64 \mu \mathrm{m}$ that coincide with appropriate absorption lines of these species having high pulse energy and average power as well as excellent spectral and spatial properties.

Within this study we have realized more than $50 \mathrm{~mJ}$ of pulse energy in the near IR coincident with appropriate absorption lines using an injection-seeded optical parametric oscillator-amplifier system pumped at $100 \mathrm{~Hz}$. At the same time this device showed excellent spectral and spatial properties. Bandwidths of less than $100 \mathrm{MHz}$ with a high degree of spectral purity $\left(>99.9 \%\right.$ ) have been achieved. The frequency stability was likewise excellent. The $\mathrm{M}^{2}$-factor was better than 2.3 .

Owing to these outstanding properties optical parametric devices are currently under investigation for the $\mathrm{CH} 4$ lidar instrument on the projected French-German climate satellite MERLIN. A similar device is under development at DLR for the lidar demonstrator CHARM-F which will enable the simultaneous measurement of $\mathrm{CO} 2$ and $\mathrm{CH} 4$ from an airborne platform.
\end{abstract}

Keywords: Spaceborne lidar, airborne lidar, greenhouse gases, carbon dioxide, methane, optical parametric oscillators, optical parametric amplifiers

\section{INTRODUCTION}

Carbon dioxide (CO2) and methane (CH4) have been recognized by the International Panel of Climate Change (IPCC) as the most important of the Earth's greenhouse gases whose concentration has been directly modified by human activities $^{[1]}$. CO2 is the single most important anthropogenic greenhouse gas in the atmosphere, contributing to $\sim 63 \%$ to the overall global radiative forcing. Its concentration has grown from a pre-industrial average mole fraction of about 280 parts per million volume (ppm) to $386 \mathrm{ppm}$ in 2008 which is an increase of $38 \%{ }^{[2]}$.

Methane has an estimated global warming potential per molecule 25 times greater than CO2 over a 100 year horizon and 72 times greater over a 20 years horizon and, despite its much lower abundance, thus is the second most significant anthropogenic greenhouse gas. $\mathrm{CH} 4$ contributes $\sim 18 \%$ to the overall global radiative forcing caused by the increase in well-mixed greenhouse gases (WMO, 2010). The increase of the average mole fraction of methane was even higher than for CO2: it rose by $158 \%$ from $\sim 700$ parts per billion volume (ppb) in the pre-industrial period to 1803 ppb in $2009^{[2]}$.

Further increase of both gases is expected to result in a warmer climate ${ }^{[1]}$. Large uncertainties in their budget, however, limit the accuracy of climate change projections. In order to reliably predict the climate of our

*Andreas.Fix@dlr.de; phone +49 8153 282577; fax 498153 281271; www.dlr.de/ipa/ 
planet, and to help constrain political conventions on greenhouse gas avoidance, adequate knowledge of the sources and sinks of these greenhouse gases and their feedbacks is mandatory. In spite of the recognized importance of this issue, our current understanding about sources and sinks of the gases $\mathrm{CO} 2$ and $\mathrm{CH} 4$ is still inadequate ${ }^{[1]}$. The $\mathrm{CO} 2$ concentration in the atmosphere is determined by the emissions from combustion of fossil fuels and by $\mathrm{CO} 2$ uptake and release by the Earth's oceans, and terrestrial biosphere. However, the rate of increase in atmospheric $\mathrm{CO} 2$ is not as high as expected based on the increase in fossil fuel emissions. This "missing" carbon is being taken up by either the oceans or the terrestrial biosphere (plants and soils), or both. Quantifying changes in $\mathrm{CH} 4$ emissions is complicated because, for example, emissions from wetlands and biomass burning are dispersed over large areas, with emission rates that vary significantly inter-annually. The biogeochemical cycles are also strongly affected by climate and land-use change. Changes in drainage resulting from changes in the distribution of permafrost are also likely to change carbon storage and release from Arctic ecosystems. Release of methane (CH4) to the atmosphere from thawing Arctic permafrost is a supposedly positive feedback to climate warming ${ }^{[3]}$.

A powerful tool to improve our knowledge of processes and rates of $\mathrm{CO} 2$ and $\mathrm{CH} 4$ exchange between atmosphere and biosphere is inverse modeling ${ }^{[4]}$. Hereby, estimates of fluxes in a chemical transport model are adjusted to provide an improved fit to observations by minimizing the errors in the estimated emissions. For this purpose more highly accurate observations with high spatial resolution and spatial coverage are required. Satellite missions have the potential to provide a denser and better distributed set of observations compared to the current network of sparse surface measurements. Thus, there is a need to develop satellite techniques for greenhouse gas observations to improve our knowledge of sources and sinks.

To address this need, a variety of studies, developments and experimental demonstrations is currently carried out. In particular, the Integrated Path Differential Absorption (IPDA) lidar technique using hard target reflection from the earth's surface in the near IR to measure the column averaged dry air mixing ratio of $\mathrm{CO} 2$ and $\mathrm{CH} 4$ with high accuracy and low bias has been found potentially suited for fulfilling the target observational requirements ${ }^{[5],[6],[7],[8],[9],[10],[11],[12]}$. The advantages of a lidar mission are that it does not require the sun as a light source, and can therefore provide both day and night, all-seasons and all latitude measurements. But more importantly, such an active mission concept provides a direct measurement of the atmospheric path and thus can assure the observation of the entire atmospheric column. This is an advantage over passive remote sensing such as SCIAMACHY on ENVISAT ${ }^{[13]}$ or TANSO-FTS on GOSAT ${ }^{[14]}$, which are particularly sensitive to the presence of aerosol.

As a mission comprising a nadir-viewing CO2 lidar instrument as the core element, A-SCOPE (Advanced Space Carbon and Climate Observation of Planet Earth) was investigated by ESA as a prephase-A Earth Explorer Mission ${ }^{[15]}$. ASCOPE did not yet advance for later phases of the Earth Explorers Core missions due to lacking technology readiness. NASA is pursuing a similar concept with ASCENDS (Active Sensing of CO2 Emissions over Nights, Days, and Seasons) ${ }^{[16]}$. Within the scope of a German-French climate monitoring initiative DLR and CNES are preparing MERLIN for launch in the 2016 time frame, a joint satellite mission with a CH4 lidar instrument onboard, which has now advanced into its phase $\mathrm{A}$.

In order to expedite the greenhouse gas lidar technology CHARM-F, an airborne demonstrator to measure both CH4 and $\mathrm{CO} 2$ columns is currently being built at DLR. A basic prerequisite, however, for a space-borne (and airborne) IPDA instrument is the availability of adequate laser sources in these wavelength regions that fulfill the challenging requirements. Since not many well characterized and mature laser materials are available for the generation of high power radiation in this wavelength region, nonlinear frequency conversion techniques such as optical parametric oscillators (OPOs) and optical parametric amplifiers (OPAs) appear favorable and have been investigated with respect to efficiency, spectral as well as spatial quality. Based on the results achieved these sources are now considered as the baseline approach for MERLIN and are currently integrated for the airborne demonstrator CHARM-F.

\section{REQUIREMENTS}

Spectral aspects of the laser transmitter play a major role in the definition of the spaceborne and airborne instruments, as the derived spectral stability and purity requirements at instrument level are extremely demanding. For CO2 IPDA two possible wavelength sets at $1.57 \mu \mathrm{m}$ and $2.05 \mu \mathrm{m}$ have been identified as suitable for measurement from space ${ }^{[18],[19],[20]}$. For CH4 IPDA wavelengths around $1.65 \mu \mathrm{m}$ show favorable properties to achieve the 
Table 1. Approximate requirements for the laser transmitter of a spaceborne IPDA lidar

\begin{tabular}{|l|c|c|c|}
\hline \multicolumn{1}{|c|}{ Parameter } & \multicolumn{2}{c|}{ CO2 } & CH4 \\
\hline Wavelength range & $1.57 \mu \mathrm{m}$ & $2.05 \mu \mathrm{m}$ & $1.64 \mu \mathrm{m}$ \\
\hline Transmitter Pulse Energy & $50 \mathrm{~mJ}$ & $50 \mathrm{~mJ}$ & $10 \mathrm{~mJ}$ \\
\hline Bandwidth & $60 \mathrm{MHz}$ & $60 \mathrm{MHz}$ & $60 \mathrm{MHz}$ \\
\hline Mean frequency stability & $70 \mathrm{kHz}$ & $270 \mathrm{kHz}$ & $10 \mathrm{MHz}$ \\
\hline Spectral Purity & $99.97 \%$ & $99.74 \%$ & $99.0 \%$ \\
\hline
\end{tabular}

measurement requirements with respect to absorption cross-section, temperature insensitivity, and optimum weighting functions ${ }^{[19],[21]}$. Several performance modeling activities have been carried out to assess the requirements for the IPDA lidar transmitters ${ }^{[19],[21],[24]}$. The basic transmitter requirements are listed in Table 1. In the context of this paper these numbers should be regarded as guidelines since the exact numbers are matter of the individual allocation of error budget.

The reason that the requirements for methane are considerably relaxed versus $\mathrm{CO} 2$ stem from the fact that anthropogenic methane sources make up $\sim 60 \%$ of the total emissions ${ }^{[3]}$, and measurement accuracy and precision need not be as rigorous as for carbon dioxide where the anthropogenic contributions are blurred by natural variability. Furthermore, the methane molecule shows a significant spectroscopic advantage over carbon dioxide since the existence of troughs in between line doublets drastically reduces the accuracy requirements of the transmitter's frequency stability ${ }^{[19],[21]}$. As a consequence, MERLIN will be a much less complex satellite mission compared to its $\mathrm{CO} 2$ counterpart.

The experimental investigations within this study were concentrated on the wavelength range at $1.57 \mu \mathrm{m}$ since here the requirements are the most stringent. Since optical parametric frequency conversion is a versatile concept, the results can be readily transferred to the other wavelengths ranges of interest. To the best of our knowledge no results on OPO/OPAs have been reported that achieved the requirements concerning spectral properties ${ }^{[22]}$ and average power ${ }^{[23]}$ at the same time.

\section{EXPERIMENTAL INVESTIGATIONS}

\subsection{Experimental Set-up}

As the pump laser for the OPO and OPA, respectively, a diode-pumped Q-switched Nd:YAG Laser (Model Spitlight DPSS INNOLAS GmbH, Germany) was used. The laser is built in form of a commonly used master-oscillator poweramplifier (MOPA) design. Single-longitudinal mode operation of the Nd:YAG laser which is essential for narrowband operation of the OPO is achieved by injection seeding. The seed source is a commercially available DFB fiber laser at $1064 \mathrm{~nm}$. The cavity length of the oscillator is controlled via the Q-switch build-up time reduction technique.

The laser generates pulse energies of $\sim 250 \mathrm{~mJ}$ at $100 \mathrm{~Hz}$ repetition rate. The beam quality factor M2 was measured to be on the order of 1.7 for both oscillator and amplifier, thus no significant degradation of the spatial beam quality is introduced in the amplification process. However, for the OPA experiments the design was improved using a partial reflecting mirror (PR) between oscillator and amplifier (see Figure 1). Approximately 15mJ of pump energy from the oscillator are extracted and were used to pump the OPO. The remaining part is amplified up to $\sim 200 \mathrm{~mJ}$ and can be used to pump the OPA. The optical path through the amplifier is long enough to compensate for the build-up time of the OPO. Thus, no extra delay line to match the onset of the OPA pump pulse is required. In order to pump the OPO a beam reduction telescope was inserted into the OPO pump beam. 


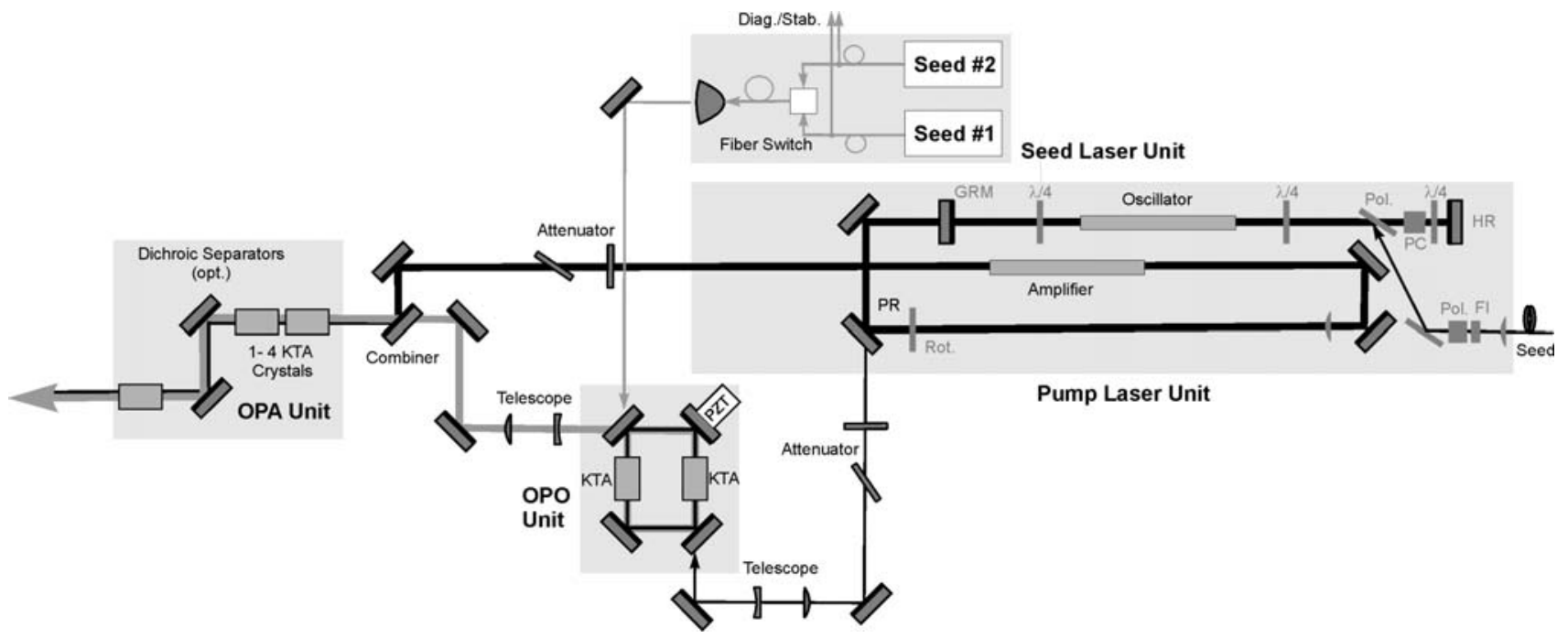

Figure 1. Set-up of the entire narrowband OPO/OPA system consisting of the Nd:YAG pump laser, the OPO unit, a seed laser unit system for narrowband operation of the OPO/OPA and an OPA stage.

The OPO set-up was chosen to be a four-mirror ring cavity with potassium titanyl arsenate (KTA) in critical phasematching cut for wavelengths around 1570nm. KTA was chosen as the nonlinear material since it has much lower absorption in the idler spectral range $(\sim 3300 \mathrm{~nm})$ compared to the often used material KTP. Two identical crystals were employed in compensated configuration to partially prevent the walk-off effect. One of the resonator mirrors is attached to a PZT element to match the cavity to the seed wavelength. The whole set-up is depicted in Figure 1.

DFB fiber lasers were used for injection seeding of the OPO. In general, two of these lasers at slightly different wavelengths (on- and off-line) are coupled to a fast fiber switch to deliver seed radiation at the selected wavelength to the OPO on a shot-to shot basis if required. The seed power available for the OPO was of the order of 5-9 $\mathrm{mW}$. The lasers were stabilized to either a $\mathrm{CO} 2$ absorption cell or to a frequency comb (Menlo Systems). Using the frequency comb as the reference, an absolute frequency stability derived from the error signal of the stabilization is of the order of $500 \mathrm{~Hz}$, only. The side mode suppression ratio of the DFB fiber seed lasers was better than $55 \mathrm{~dB}$.

In order to stably seed the OPO a cavity stabilization technique is necessary to match the cavity length to the seed wavelength. For this purpose, a stabilization concept has been implemented based on the experience from prior work ${ }^{[25],[26]}$. This concept is based on the measurement of the frequency difference between the outgoing OPO pulse and the incoming cw seed radiation on a shot-by-shot basis. This is done by mixing part of the seed light which is acoustooptically frequency shifted by $\sim 500 \mathrm{MHz}$ with the outgoing OPO pulse by using a fast (bandwidth $\sim 2 \mathrm{GHz}$ ) photo diode. For each pulse, the beat signal is digitized by an $8 \mathrm{bit} / 2 \mathrm{GHz}$ transient recorder and its power spectrum is calculated by fast Fourier transform (FFT). The error signal for the loop control is generated from the difference between the two frequencies.

The advantage of this stabilization scheme is that next to generating the control signal from the frequency difference the power spectrum and thus both measured centre frequency and bandwidth of the OPO is available for each individual pulse. It also shows advantages to other commonly used stabilization techniques since no sharp resonance structures are required to achieve the necessary stability requirements. A disadvantage of the stabilization scheme used for the OPO is that it is not possible to react to vibrations with frequencies exceeding half the repetition rate of $50 \mathrm{~Hz}$. However, the OPO cavity is a small ring milled from a monolithic aluminum block and shows a rather good short-term stability. Results from the characterization of the stabilization loop are given below.

By means of a telescope the OPO signal beam was magnified to match the diameter of the OPA pump. Using a dichroic combiner, OPO signal and Nd:YAG beam whose energy could be varied by means of a variable attenuator were superimposed to enter the OPA stage. Following the pioneering investigation of Arisholm et al. ${ }^{[22]}$ we investigated multi-crystal systems consisting of 1-4 KTA crystals of 15-mm length each and compared linear setups with set-ups that contained dielectric separators to eliminate the idler radiation between selected crystals. According to that study it is expected that this can improve the beam profile. 

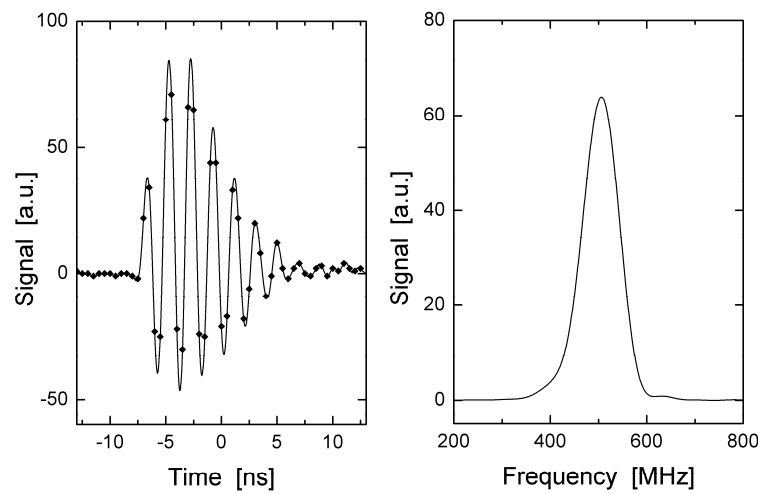

Figure 2. Signals from the stabilization. Left: Beat spectrum. The diamonds depict the measurement points and the line is the fitted beat spectrum to the heterodyne measurement. Right: Power spectrum derived from the beat signal. The centre frequency is $\sim 500 \mathrm{MHz}$ due to the frequency shift of the AOM. The width (FWHM) of this spectrum is $88 \pm 1 \mathrm{MHz}$.

\subsection{Optical Parametric Oscillator}

In seeded operation the maximum output energy of the seeded OPO was $4.15 \mathrm{~mJ}$ and $3.40 \mathrm{~mJ}$ for the unseeded OPO, respectively (Figure 3). The comparison of output energy versus the number of times above threshold shows that the slope efficiency in both cases is very comparable, nevertheless.

The pulse width of the OPO was measured to be $6 \mathrm{~ns}$. This is anticipated since the pulse width of an OPO is generally shorter than that of the pump ( $\sim 8 \mathrm{~ns})$. Due to the OPO build-up time the onset of the OPO pulse is delayed with respect to its pump pulse. Concerning the timing of OPA pump and OPO the chosen set-up with the partial reflector between $\mathrm{Nd}$ :YAG oscillator and amplifier shows the expected advantage that no delay line between of the amplified pump is necessary, indeed.

By using the technique of injection seeding bandwidths of the order of $90 \mathrm{MHz}$ were achieved. Although this is somewhat broader than required this is purely dependent on the pulse width of the pump laser. Longer pulses that were not accessible within the scope of this study will enable further reduction of the spectral width of the OPO which is already close $(\sim 1.2 \mathrm{x})$ to the Fourier limit.
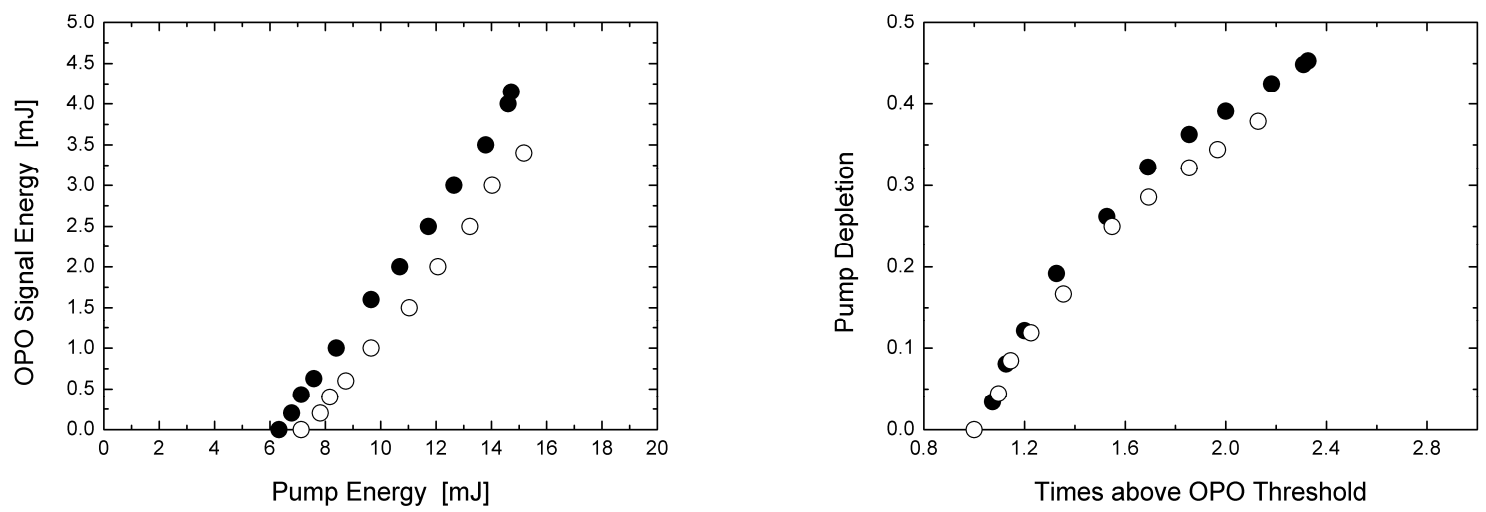

Figure 3. Left: Signal energy of the OPO at $1572 \mathrm{~nm}$ as function of pump energy at $1064 \mathrm{~nm}$ when the OPO is seeded (full circles) and unseeded (open circles). Right: Measured depletion of the pump as function of times above OPO. 

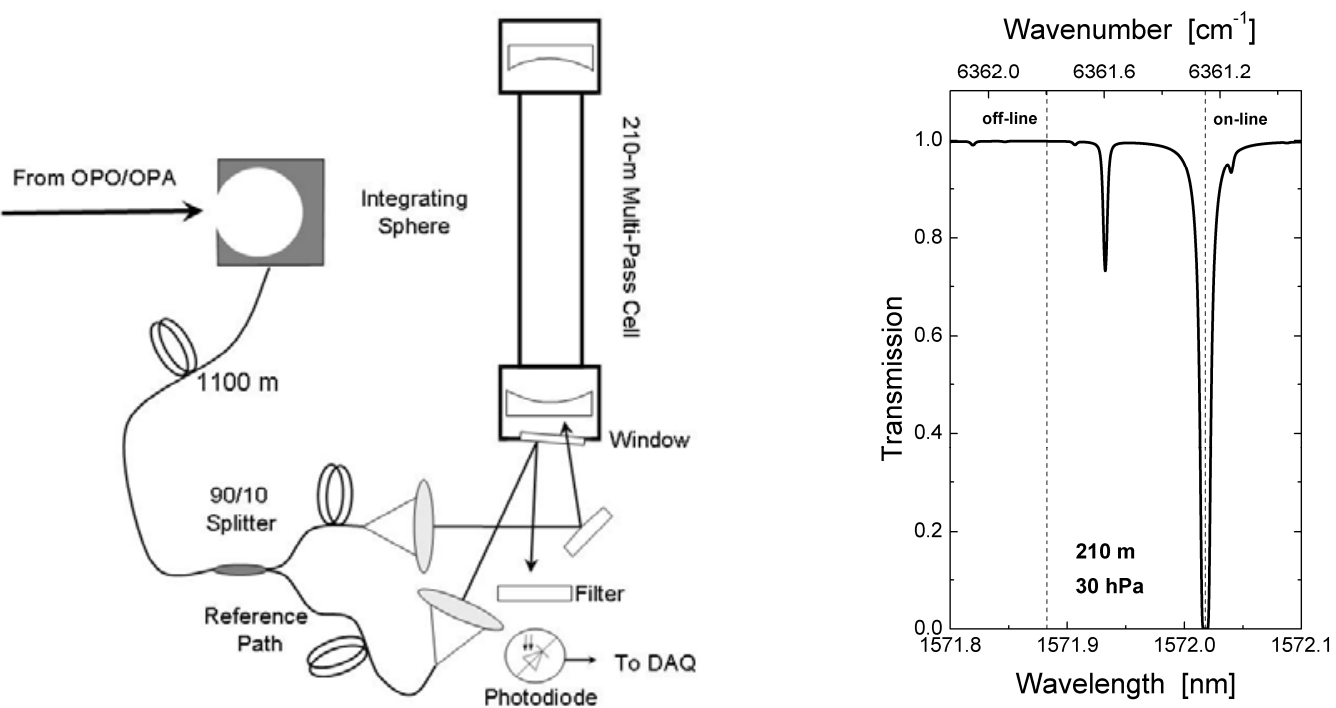

Figure 4. Left: Set-up to measure the spectral purity of OPO and OPA, respectively. The multipass absorption with a path length of $210 \mathrm{~m}$ was filled with $30 \mathrm{hPa}$ of CO2. The spectrum according to HITRAN 2008 is depicted on the right together with the positions of the on- and off-line wavelengths (dashed lines). Theoretically, the transmission at the on-line wavelength should be less than $10^{-6}$.

The seeded OPO when stabilized by means of the above described heterodyne technique showed an Allan deviation of $<$ $70 \mathrm{KHz}$ relative to the seed source for averaging times of more than $3 \mathrm{~s}$. This figure depicts the OPO's absolute stability since the absolute stability of the seed is orders of magnitude better $(\sim 500 \mathrm{~Hz})$.

The spectral purity (SP) of the OPO (which is defined as the ratio of the energy contained in a narrow spectral interval typically the width of the absorption line to the total energy) was measured using a multipass absorption cell. The accurate determination of a fraction of a percent of broadband light present in the laser beam makes it a challenging task. A convenient way to measure the spectral purity is the use of long-pass absorption cells ${ }^{[27],[28],[29]}$. This requires, however, that the transmission $\mathrm{T}$ of the cell is $\mathrm{T}<1$-SP.

For the spectral purity measurements within the scope of this study we therefore used a multi-pass cell with an optical path length of $210 \mathrm{~m}$ filled with pure $\mathrm{CO}_{2}$ typically at pressures of $\sim 30 \mathrm{hPa}$ (see Figure 4). For convenience, a multimode optical fiber was used to propagate the OPO radiation from the measurement point to the cell. To sample the full beam an integrating sphere was inserted into the OPO beam. The fiber had a geometrical length of $1100 \mathrm{~m}$ which leads to a temporal separation of signal and electronic noise introduced by the laser. In addition, a fiber splitter was introduced to realize a reference path by which the power onto the cell can be monitored. By means of an output coupler part of the reference beam was reflected off the tilted cell window and directed onto the detector. An interference filter with a 10$\mathrm{nm}$ wide bandpass is used to suppress ambient light. The whole measurement set-up is depicted in Figure 4. Usually about 5000 individual pulses had to be averaged to be able to distinguish any spectral impurity from background noise. By means of injection seeding a degree of the spectral purity of better than $99.98 \%$ was achieved even for seed powers in the range of $150 \mu \mathrm{W}$ (see Figure 5).

Finally, the spatial beam quality of the OPO was determined using an InGaAs camera. According to ISO 11146 the beam quality factor M2 was measured to be of the order of 2.0. 

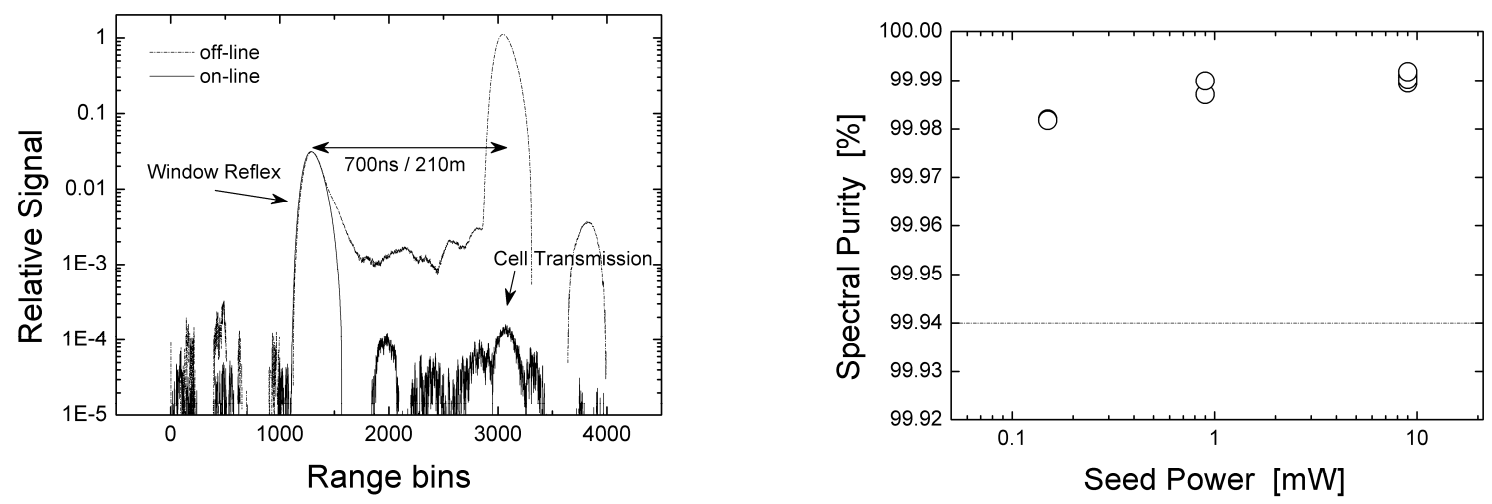

Figure 5. Left: On-line (solid) and off-line (dashed) signals behind the multipass absorption cell. The window reflex is clearly seen at $\sim 1200$ range bins. The transmitted signal is delayed by $700 \mathrm{~ns}$. Off-line and on-line pulses are normalized so that they show the same peak height of the window reflex pulse. In this case the seed power was 0.9 $\mathrm{mW}$. From this measurement the spectral purity is derived to be $99.987 \%$. The right graph shows the result for various seed powers in the range from $0.15-9 \mathrm{~mW}$. Even for a seed power as low as $150 \mu \mathrm{W}$ a spectral purity of better than $99.98 \%$ was achieved.

\subsection{Optical Parametric Amplifier}

Several multi-crystal OPA configurations were compared to yield optimum conversion efficiency, and best spatial beam profile. It turned out that for the given configuration four crystals already led to back conversion so that three-crystal setups are to be preferred.

Figure 6 shows the OPA signal energy of two configurations in comparison. Both configurations do not show significant differences in output energy nor gain. In summary, a maximum OPA signal energy of $54.8 \mathrm{~mJ}$ (5.5 W of average power) was achieved at a pump energy of $192 \mathrm{~mJ}(19.2 \mathrm{~W}$ of average power). In addition, a maximum gain of 18 and a maximum pump depletion of as high as $38.5 \%$ were determined.
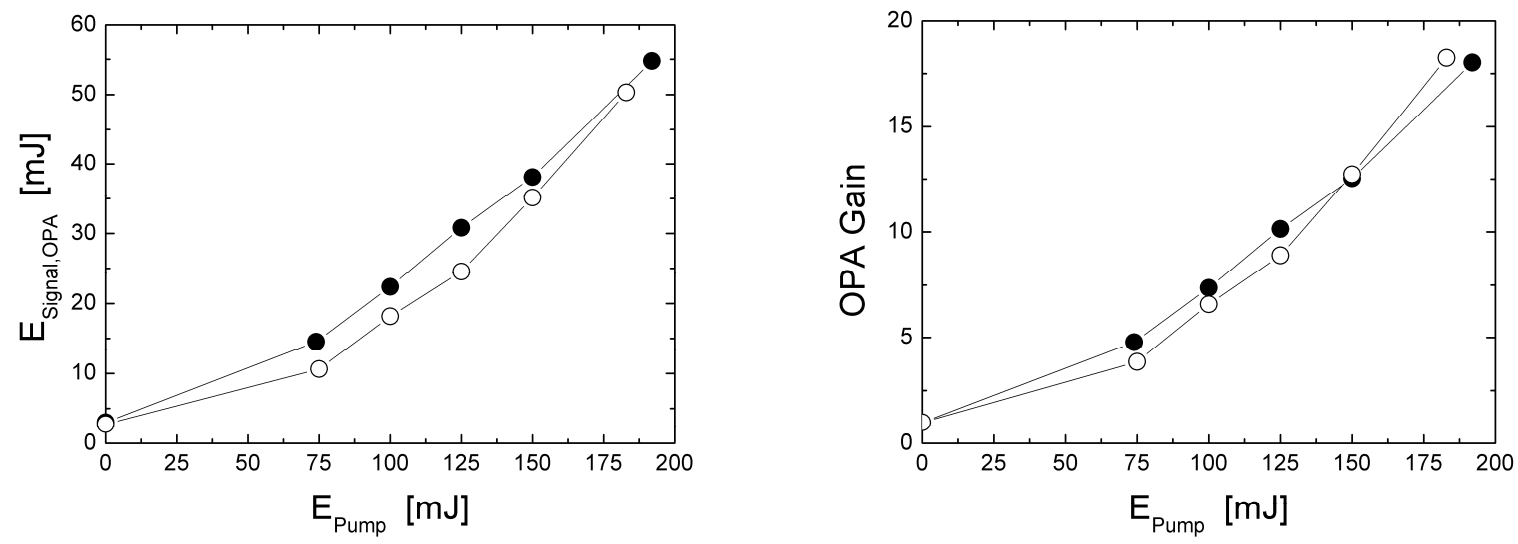

Figure 6. Left: OPA signal energy as function of pump energy for two different three-crystal configurations. The solid dots represent the case of a linear amplifier stage without separation of the idler beam. The open dots represent the case where the idler is removed between the second and third amplifier crystal. Right: Gain of the two amplifier configurations as function of OPA pump energy. 
According to the study of Arisholm et al. ${ }^{[22]}$ it is expected that the beam profile is better for the configuration with idler separation. This could be confirmed although the difference is not big. The OPA beam profile showed $\mathrm{M}^{2}$-values of 2 (for the configuration with idler separation) and 2.3, respectively, which is remarkably good compared to the input beams of OPO signal $\left(\mathrm{M}^{2} \sim 2.0\right)$ and Nd:YAG pump $\left(\mathrm{M}^{2} \sim 1.7\right)$. The spectral purity of the OPA was found to be slightly worse than the oscillator but still better than $99.90 \%$. As a result of a careful analysis it is believed that this reduced spectral purity is due to insufficient spectral properties of the pump laser.

\section{CONCLUSIONS}

To summarize, an injection-seeded optical parametric oscillator/optical parametric amplifier (OPO/OPA) system pumped at the fundamental of a diode-pumped $\mathrm{Nd}: \mathrm{YAG}$ laser at $100 \mathrm{~Hz}$ repetition frequency has been devised.

Following a careful selection of crystals, a system was devised based on potassium titanyl arsenate (KTA) as the nonlinear material for both OPO and OPA to generate the required wavelengths at appropriate absorption lines of CO2 near $1572 \mathrm{~nm}$. Several configurations have been analyzed with respect to energy and efficiency as well as spatial and spectral properties.

The main requirements for a CO2 IPDA transmitter could be achieved. By means of two OPA configurations consisting of three amplifier crystals output energies of more than $50 \mathrm{~mJ}$ at $1572 \mathrm{~nm}$ could be generated. This corresponds to average powers of about $5.5 \mathrm{~W}$. The pump energy needed for the amplifier was on the order of $185 \mathrm{~mJ}$. For the OPO a pump energy of $15 \mathrm{~mJ}$ was utilized to generate an OPO signal energy on the order of $3 \mathrm{~mJ}$. Therefore, a pump energy of $200 \mathrm{~mJ}$ was required in total. Thus, the total efficiency with respect to the signal at $1572 \mathrm{~nm}$ exceeds $25 \%$.

In addition, the spatial beam quality of the OPA was found to be very satisfying. Starting from the pump laser which, due to its unstable resonator architecture, has an M2-value of $\sim 1.7$ the OPO signal beam had a M2 $\sim 2$. The amplifier did not deteriorate this beam significantly. At full energy its M2-value was less than 2.3. The OPO when seeded with a fiber laser stabilized to a frequency comb showed a absolute stabilization of its center frequency of $<70 \mathrm{kHz}$ (expressed as the Allan deviation for averaging times of more than $3 \mathrm{~s}$ ).

To the best of our knowledge, this study represents the first investigation of a pulsed, injection-seeded OPO that is amplified by means of an OPA to average powers beyond $5 \mathrm{~W}$. Other studies operated OPOs broadband ${ }^{[2]}$ or generated narrowband OPO operation by means of different techniques ${ }^{[23]}$. Neither of these achieved output powers in the range reported here. In addition, this is the first study using KTA as the OPA material we are aware of.

In summary, OPO/OPA systems when pumped with the fundamental of a Nd:YAG laser and injection seeded to provide narrowband operation appear to be very promising candidates as appropriate transmitters for an IPDA lidar to measure $\mathrm{CO} 2$ or $\mathrm{CH} 4$ from space at $1.57 \mu \mathrm{m}, 1.64 \mu \mathrm{m}$, or at $2.05 \mu \mathrm{m}$, respectively. They are now considered as the baseline approach for the German-French methane satellite mission MERLIN and the airborne demonstrator CHARM-F to be flown on the German Research aircraft HALO.

\section{ACKNOWLEDGMENTS}

This work received funding from European Space Agency under contract no. 18256/04/NL/EF. We are grateful to P. Zeller, Astrium GmbH, for his support.

\section{REFERENCES}

[1] Solomon, S., D. Qin, M. Manning, Z. Chen, M. Marquis, K.B. Averyt, M. Tignor and H.L. Miller (eds.), "Contribution of Working Group I to the Fourth Assessment Report of the Intergovernmental Panel on Climate Change," Cambridge University Press 2007.

[2] WMO World Data Centre for Greenhouse Gases, WDCGG Data Summary No. 35, March 2011.

[3] Heimann, M., "How Stable Is the Methane Cycle?" Science, 327, 1211-1212 (2010). 
[4] Gurney, K.R., R.M. Law, A.S. Denning, P.J. Rayner, D. Baker, P. Bousquet, L. Bruhwiler, Y.H. Chen, P. Ciais, S. Fan, I.Y. Fung, M. Gloor, M. Heimann, K. Higuchi, J. John, T. Maki, S. Maksyutov, K. Masarie, P. Peylin, M. Prather, B.C. Pak, J. Randerson, J. Sarmiento, S. Taguchi, T. Takahashi, and C.W. Yuen, "Towards robust regional estimates of CO2 sources and sinks using atmospheric transport models," Nature 415, 626-630 (2002).

[5] Koch G.J., J. Y. Beyon, F. Gibert, B.W. Barnes, S. Ismail, M. Petros, P. J. Petzar, J. Yu, E. A. Modlin, K. J. Davis, and U. N. Singh, "Side-line tunable laser transmitter for differential absorption lidar measurements of CO2: design and application to atmospheric measurements," Appl. Opt. 47, 944-956 (2008).

[6] Kameyama S., M. Imaki, Y. Hirano, S. Ueno, S. Kawakami, D. Sakaizawa, and M.Nakajima, "Development of $1.6 \mu \mathrm{m}$ continuous-wave modulation hard-target differential absorption lidar system for CO2 sensing," Opt. Lett. 34, 1513-1515 (2009).

[7] Browell, E. V., J. Dobler, S. Kooi, Y. Choi, F. Harrison, B. Moore, T. Zaccheo, ”Airborne validation of active CO2 LAS measurements," EOS, Trans. Am. Geophys. Un. 90(52), Fall Meet. Suppl., (2009).

[8] Amediek, A., Fix, A., Wirth, M. and Ehret, G., "Development of an OPO system at $1.57 \mu \mathrm{m}$ for integrated path DIAL measurement of atmospheric carbon dioxide", Appl. Phys. B 92, 295-302 (2008).

[9] Kawa S. R., J. Mao, J. B. Abshire, G. J. Collatz, X. Sun, and C. J. Weaver, "Simulation studies for a spacebased CO2 lidar mission," Tellus B 62, 759-769 (2010).

[10] Abshire J. B., H. Riris, G. R. Allan, C. J. Weaver, J. Mao, Z. Sun, W. E. Hasselbrack, S. R. Kawa, and S. Biraud, "Pulsed airborne lidar measurements of atmospheric CO2 column absorption," Tellus 62B, 770-783 (2010).

[11] Spiers G. D., R. T. Menzies, J. Jacob, L. E. Christensen, M. W. Phillips, Y. Choi, and E. V. Browell, "Atmospheric CO2 measurements with a 2- $\mu \mathrm{m}$ airborne laser absorption spectrometer employing coherent detection," Appl. Opt. 50, 2098-2111 (2011).

[12] Kameyama S., M. Imaki, Y. Hirano, S. Ueno, S. Kawakami, D. Sakaizawa, T. Kimura, and M. Nakajima, "Feasibility study on $1.6-\mu \mathrm{m}$ continuous-wave modulation laser absorption spectrometer system for measurement of global CO2 concentration from a satellite," Appl. Opt. 50, 2055-2068 (2011).

[13] Schneising, O., M. Buchwitz, M. Reuter, J. Heymann, H. Bovensmann, and J. P. Burrows, "Long-term analysis of carbon dioxide and methane column-averaged mole fractions retrieved from SCIAMACHY," Atmos. Chem. Phys., 11, 2863-2880 (2011).

[14] Yokota T., Y. Yoshida, N. Eguchi, Y. Ota, T. Tanaka, H. Watanabe and S. Maksyutov; "Global Concentrations of CO2 and CH4 Retrieved from GOSAT: First Preliminary Results”, SOLA, Vol. 5, pp.160-163 (2009) .

[15]ESA, “A-SCOPE, Advanced space carbon and climate observation of planet earth,” Report for Assessment, SP1313/1, ESA communication production office, Noordwijk, The Netherlands, 2008. ISBN 978-92-9221-406-7.

[16] Michalak, A., C. Miller, E. Browell, B. Moore, J. Abshire, and co-authors, "ASCENDS—active Sensing of CO2 Emissions over Nights, Days, and Seasons Mission," Technical report, University of Michigan in Ann Arbor, Michigan 2008.

[17] Bosenberg W. R., and D. R. Guyer, "Broadly tunable, single-frequency optical parametric frequency-conversion system," J. Opt. Soc. Am. B 10, 1716-1722 (1993).

[18] Menzies R. T. and D. M. Tratt, "Differential laser absorption spectrometry for global profiling of tropospheric carbon dioxide: selection of optimum sounding frequencies for high-precision measurements," Appl. Opt. 42, 6569-6577 (2003).

[19] Ehret, G., Kiemle, C., Wirth, M., Amediek, A., Fix, A., Houweling, S., "Space-borne remote sensing of CO2, $\mathrm{CH} 4$, and $\mathrm{N} 2 \mathrm{O}$ by integrated path differential absorption lidar: a sensitivity analysis", Applied Physics B: Lasers and Optics 90, 593-608 (2008).

[20]Caron J., and Yannig Durand, "Operating wavelengths optimization for a spaceborne lidar measuring atmospheric CO2," Appl. Opt. 48, 5413-5422 (2009).

[21] Kiemle C., M. Quatrevalet, G. Ehret, A. Amediek, A. Fix, and M. Wirth, "Sensitivity studies for a space-based methane lidar mission," Atmos. Meas. Tech. Discuss., 4, 3545-3592 (2011).

[22] Arisholm G., Ø. Nordseth, and G. Rustad, "Optical parametric master oscillator and power amplifier for efficient conversion of high-energy pulses with high beam quality,"Opt. Express 12, 4189-4197 (2004).

[23] Raybaut M., T. Schmid, A. Godard, A. K. Mohamed, M. Lefebvre, F. Marnas, P. Flamant, A. Bohman, P. Geiser, and P. Kaspersen, "High-energy single-longitudinal mode nearly diffraction-limited optical parametric source with $3 \mathrm{MHz}$ frequency stability for CO2 DIAL," Opt. Lett. 34, 2069-2071 (2009).

[24] Caron J., Y. Durand, J.-L. Bezy and R. Meynart, "Performance modeling for A-SCOPE: a space-borne lidar measuring atmospheric CO2", Proc. SPIE 7479, 74790E (2009). 
[25] Mahnke P., and M. Wirth, "Real-time quantitative measurement of the mode beating of an injection-seeded optical parametric oscillator," Appl. Phys. B 99, 141-148 (2010).

[26] Wirth M., A. Fix, P. Mahnke, H. Schwarzer, F. Schrandt and G. Ehret, "The airborne multi-wavelength water vapor differential absorption lidar WALES: system design and performance," Appl. Phys. B96, 201 (2009).

[27] Ponsardin, P., N. S. Higdon, B. E. Grossmann, and E. V. Browell, "Spectral control of an alexandrite laser for an airborne water-vapor differential absorption lidar system," Appl. Opt. 33, 6439-6450 (1994).

[28] Poberaj, G., A. Fix, A. Assion, M. Wirth, C. Kiemle, and G. Ehret, "Airborne all-solid-state DIAL for water vapour measurements in the tropopause region: system description and assessment of accuracy," Appl. Phys. B $75,165-172$ (2002).

[29] Mahnke, P., H.H. Klingenberg, A. Fix, and M. Wirth, "Dependency of injection seeding and spectral purity of a single resonant KTP optical parametric oscillator on the phase matching condition," Appl. Phys. B 89, 1-7 (2007). 